JOURNAL OF

SYMPLECTIC GEOMETRY

Volume 3, Number 4, 655-670, 2005

\title{
MOMENT MAPS, SYMPLECTOMORPHISM GROUPS AND COMPATIBLE COMPLEX STRUCTURES
}

\author{
Miguel Abreu, Gustavo Granja, and Nitu Kitchloo
}

In this paper, we apply Donaldson's general moment map framework for the action of a symplectomorphism group on the corresponding space of compatible (almost) complex structures to the case of rational ruled surfaces. This gives a new approach to understanding the topology of their symplectomorphism groups, based on a result of independent interest: the space of compatible integrable complex structures on any symplectic rational ruled surface is (weakly) contractible. We also explain how in general, under this condition, there is a direct relationship between the topology of a symplectomorphism group, the deformation theory of compatible complex structures and the groups of complex automorphisms of these complex structures.

\section{Introduction}

The known results regarding the topology of symplectomorphism groups, for two-dimensional surfaces and four-dimensional rational ruled surfaces, indicate a direct relation with the topology of the corresponding groups of complex automorphisms. The goal of this paper is to formulate this empirical relation more precisely, within a general framework involving infinite dimensional groups, manifolds and moment maps. This general framework goes back to Atiyah and Bott [5], was made rigorous and precise in finite dimensions by Kirwan [18] and was shown to apply to the action of a symplectomorphism group on the corresponding space of compatible (almost) complex structures by Donaldson [10].

It is usually quite difficult to make this general framework rigorous and precise in infinite dimensions, and we will make no attempt at that here. However, its formal application is often very useful as a guide to the results one should expect and to the approach one might take in proving them. We will present here two results, Theorems 3.5 and 3.6, that are an example of this. Their rigorous proofs will appear in [2]. 
The paper is organized as follows. In Section 2, we describe the general moment map framework and how it applies to the action of a symplectomorphism group on the corresponding space of compatible (almost) complex structures. In Section 3, we discuss the particular case of rational ruled surfaces, where the geometric picture suggested by the moment map framework is quite accurate and gives a new approach to understanding the topology of their symplectomorphism groups. This approach is based on the fact that the space of compatible integrable complex structures on any symplectic rational ruled surface is (weakly) contractible (Theorem 3.5). Any time this condition holds, there should be a direct relationship between the problem of understanding the topology of the symplectomorphism group and the following two problems: understanding the deformation theory of compatible complex structures and understanding the topology of the groups of complex automorphisms of these complex structures. This general relation is explained in Section 4.

\section{Moment map geometry}

In this section, following [10], we recall a general moment map framework and how it applies to the action of a symplectomorphism group on the corresponding space of compatible almost-complex structures.

General framework. Let $G$ be a Lie group, $\mathcal{G}$ its Lie algebra, $\langle\cdot, \cdot\rangle$ an inner product on $\mathcal{G}$ invariant under the adjoint action, $\mathcal{G}^{*}$ the dual Lie algebra naturally identified with $\mathcal{G}$ via $\langle\cdot, \cdot\rangle$ and $G^{\mathbb{C}}$ a complexification of $G$.

Let $(X, J, \Omega)$ be a Kähler manifold equipped with an action of $G$ by Kähler isometries, i.e., a homomorphism

$$
\rho: G \longrightarrow \operatorname{Iso}(X, J, \Omega)=\operatorname{Hol}(X, J) \cap \operatorname{Symp}(X, \Omega) .
$$

Suppose this action satisfies the following two conditions:

(i) the holomorphic action of $G$ on $(X, J)$ extends to a holomorphic action of $G^{\mathbb{C}}$ on $(X, J)$;

(ii) the symplectic action of $G$ on $(X, \Omega)$ admits a suitably normalized equivariant moment map $\mu: X \rightarrow \mathcal{G}^{*}$.

Then we have the following two general principles.

General Principle I. The complex and symplectic quotients of $X$ by $G$ are naturally identified. More precisely,

$$
\frac{\mu^{-1}(0)}{G}=\frac{X^{s}}{G^{\mathbb{C}}}
$$

where $X^{s} \subset X$ is an open, $G^{\mathbb{C}}$-invariant, subset of "stable points." We will not define here this notion of stability, the important point being that it should only depend on the holomorphic geometry of the situation. The content of this principle is that on each stable $G^{\mathbb{C}}$-orbit, there is a point $p \in \mu^{-1}(0)$, unique up to the action of $G$. 
General Principle II. The map $\|\mu\|^{2} \equiv\langle\mu, \mu\rangle: X \rightarrow \mathbb{R}$ behaves like a $G$-invariant Morse-Bott function, whose critical manifolds compute the equivariant cohomology $H_{G}^{*}(X) \equiv H^{*}\left(X \times_{G} E G\right.$ ) (over $\mathbb{Q}$ and, in good special cases, also over $\mathbb{Z}$ ).

Combining these two general principles, one gets the following geometric picture for the action of $G$ on $X$ :

- The gradient flow of $-\|\mu\|^{2}$ induces an invariant stratification

$$
X=V_{0} \sqcup V_{1} \sqcup V_{2} \sqcup \cdots,
$$

where each $V_{k}$ is the stable manifold of some critical set $C_{k}$ of $\|\mu\|^{2}$.

- Let $\mathcal{O}_{k}$ denote the coadjoint orbit $G \cdot \xi_{k} \subset \mathcal{G}^{*}$, where $\xi_{k}=\mu\left(p_{k}\right)$ for some $p_{k} \in C_{k}$. Then

$$
\frac{V_{k}}{G^{\mathbb{C}}} \simeq \frac{\mu^{-1}\left(\mathcal{O}_{k}\right)}{G}
$$

If $C_{0}=\mu^{-1}(0)$, then $\mathcal{O}_{0}=\{0\}$ and $V_{0}=X^{s}$.

- The equivariant cohomology $H_{G}^{*}(X)$ can be computed from $H_{G}^{*}\left(V_{k}\right)$, $k=0,1,2, \ldots($ over $\mathbb{Q}$ and, in good special cases, also over $\mathbb{Z})$.

Symplectomorphism groups and compatible complex structures. Consider a compact symplectic manifold $(M, \omega)$, of dimension $2 n$ and assume that $H^{1}(M, \mathbb{R})=0$. Let $G \equiv \operatorname{Symp}(M, \omega)$ be the symplectomorphism group of $(M, \omega)$. This is an infinite dimensional Lie group whose Lie algebra $\mathcal{G}$ can be identified with the space of functions on $M$ with integral zero:

$$
\mathcal{G}=C_{0}^{\infty}(M) \equiv\left\{f: M \longrightarrow \mathbb{R}: \int_{M} f \frac{\omega^{n}}{n !}=0\right\} .
$$

$\mathcal{G}$ has a natural invariant inner product $\langle\cdot, \cdot\rangle$ given by

$$
\langle f, g\rangle \equiv \int_{M} f \cdot g \frac{\omega^{n}}{n !},
$$

which will be used to identify $\mathcal{G}^{*}$ with $\mathcal{G}$.

Consider now the space $\mathcal{J}(M, \omega)$ of almost complex structures $J$ on $M$, which are compatible with $\omega$, i.e., for which the bilinear form

$$
g_{J}(\cdot, \cdot)=\omega(\cdot, J \cdot)
$$

is a Riemannian metric on $M$. This is the space of sections of a bundle over $M$ with fiber the contractible symmetric Kähler manifold $S p(2 n, \mathbb{R}) / U(n) \equiv$ Siegel upper half space [24]. This fiberwise symmetric Kähler structure, together with the volume form induced by $\omega$ on $M$, turns $\mathcal{J}(M, \omega)$ into an infinite dimensional (contractible) Kähler manifold.

The symplectomorphism group $G$ acts naturally on $\mathcal{J}(M, \omega)$ by Kähler isometries:

$$
\phi \cdot J \equiv \phi_{*}(J)=d \phi \circ J \circ d \phi^{-1}, \quad \forall \phi \in G, \quad J \in \mathcal{J}(M, \omega) .
$$


To fit the previous general framework, this action should satisfy conditions (i) and (ii).

The first (holomorphic) condition poses an immediate problem since there is no complexification $G^{\mathbb{C}}$ of the symplectomorphism group $G$. However, we can certainly complexify the Lie algebra $\mathcal{G}$ to

$$
\mathcal{G}^{\mathbb{C}} \equiv\left\{f: M \longrightarrow \mathbb{C}: \int_{M} f \frac{\omega^{n}}{n !}=0\right\}
$$

and the infinitesimal action of $\mathcal{G}$ on $\mathcal{J}(M, \omega)$ extends to an action of $\mathcal{G}^{\mathbb{C}}$, since the complex structure on $\mathcal{J}(M, \omega)$ is integrable. This gives rise to an integrable complex distribution on $\mathcal{J}(M, \omega)$ whose leaves play the role of "connected components of orbits of the group $G^{\mathbb{C}}$."

In the holomorphic side of General Principles I and II that we want to apply, $G^{\mathbb{C}}$ is not that important when compared with the role played

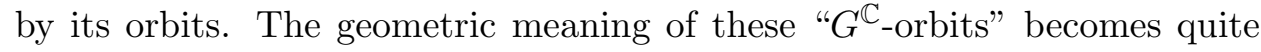
clear if one restricts the actions under consideration to the invariant Kähler submanifold $X$ of compatible integrable complex structures

$$
X \equiv \mathcal{J}^{\text {int }}(M, \omega) \subset \mathcal{J}(M, \omega),
$$

determined by the vanishing of the Nijenhuis tensor. Here, it follows from Donaldon's analysis in [10] that

$J, J^{\prime} \in X$ belong to the same " $G^{\mathbb{C}}$-orbit" iff there exists $\varphi \in \operatorname{Diff}(M)$ such that

$$
\left[\varphi^{*}(\omega)\right]=[\omega] \in H^{2}(M, \mathbb{R}) \quad \text { and } \quad \varphi_{*}(J)=J^{\prime} .
$$

This explicit description of a " $G^{\mathbb{C}}$-orbit" is good enough to consider that the holomorphic action of $G=\operatorname{Symp}(M, \omega)$ on $X=\mathcal{J}^{\text {int }}(M, \omega)$ satisfies condition (i).

Regarding condition (ii), Donaldson [10] shows that there always exists an equivariant and suitably normalized moment map

$$
\mu: \mathcal{J}(M, \omega) \longrightarrow \mathcal{G}^{*} \cong C_{0}^{\infty}(M)
$$

for the symplectic action of $G$ on $\mathcal{J}(M, \omega)$ given by

$$
\mu(J)=\left(\text { Hermitian scalar curvature } S(J) \text { of the metric } g_{J}\right)-d,
$$

where $d$ is the constant defined by

$$
d \cdot \int_{M} \frac{\omega^{n}}{n !} \equiv 2 \pi c_{1}(M) \wedge[\omega]^{n-1}(M)=\int_{M} S(J) \frac{\omega^{n}}{n !} .
$$

Note that on $X \subset \mathcal{J}(M, \omega)$, i.e., for integrable $J$, the Hermitian scalar curvature $S(J)$ coincides with the usual scalar curvature of the Riemannian metric $g_{J}$.

We have concluded that the Kähler action of $G$ on $X$ satisfies conditions (i) and (ii) of the general framework, and so General Principles I and II should apply. What do they say in this context? 
General Principle I says that each stable compatible complex structure is diffeomorphic to one in $\mu^{-1}(0)$, unique up to the action of $G$. Since

$$
J \in \mu^{-1}(0) \Longleftrightarrow \mu(J)=0 \Longleftrightarrow S(J)=d=\text { constant }
$$

this says that on each diffeomorphism class of stable compatible complex structures there should exist a unique $\operatorname{Symp}(M, \omega)$-orbit whose corresponding Kähler metric has constant scalar curvature. (See the work of Donaldson [11-13], exploring this consequence of general principle I.)

General Principle II says that the critical points of

$$
\|\mu\|^{2}: X=\mathcal{J}^{\text {int }}(M, \omega) \longrightarrow \mathbb{R}, \quad\|\mu\|^{2}(J)=\int_{M} S^{2}(J) \frac{\omega^{n}}{n !}+\text { constant },
$$

determine the equivariant cohomology $H_{G}^{*}(X)$.

These critical points are, in particular, extremal Kähler metrics in the sense of Calabi $[\mathbf{6}, \mathbf{7}]$. When extremal Kähler metrics exist, they minimize $\|\mu\|^{2}$ on the corresponding " $G{ }^{\mathbb{C}}$-orbit" $[\mathbf{1 6}]$ and are conjecturally unique up to the action of $G[\mathbf{8}]$.

In the concrete examples, we will discuss (Riemann surfaces and rational ruled surfaces) these general principles do hold. Whenever this is the case, one gets the following geometric picture for the action of $G=\operatorname{Symp}(M, \omega)$ on $X=\mathcal{J}^{\text {int }}(M, \omega)$ :

- There is a stratification of $X$ of the form

$$
X=V_{0} \sqcup V_{1} \sqcup V_{2} \sqcup \cdots \text {. }
$$

In this stratification, each $V_{k}$ contains the set of compatible complex structures which are diffeomorphic to an extremal one with normalized scalar curvature in

$$
\mathcal{O}_{k} \equiv \text { coadjoint orbit } G \cdot\left(S\left(J_{k}\right)-d\right) \text { in } C_{0}^{\infty}(M) \cong \mathcal{G}^{*},
$$

where $J_{k}$ is some critical compatible complex structure in $V_{k}$.

- " $V_{k} / G^{\mathbb{C} "} \equiv\left\{\right.$ " $G{ }^{\mathbb{C}}$-orbits" in $\left.V_{k}\right\} \simeq \mu^{-1}\left(\mathcal{O}_{k}\right) / G$ is some moduli space of complex structures that one might try to understand using methods from complex geometry (deformation theory).

- Let $\mathcal{O}_{J_{k}}$ denote the " $G$ C -orbit" through some extremal $J_{k} \in V_{k}$ and

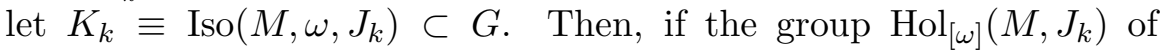
holomorphic automorphisms which preserve the cohomology class of $\omega$ is the complexification of $K_{k}$ (this is always the case if the groups are connected by $[\mathbf{7}]$ ), then the inclusion

$$
\frac{G}{K_{k}} \cong G \cdot J_{k} \hookrightarrow \mathcal{O}_{J_{k}}=\frac{" G G^{\mathbb{C} "}}{\operatorname{Hol}_{[\omega]}\left(M, J_{k}\right)}
$$

is a homotopy equivalence. 
- The equivariant cohomology $H_{G}^{*}(X)$ can be computed from $H_{G}^{*}\left(V_{k}\right)$, $k=0,1,2, \ldots$ From the previous two points, each $H_{G}^{*}\left(V_{k}\right)$ should be determined from finite dimensional considerations involving moduli spaces of complex structures and subgroups of isometries in $G=\operatorname{Symp}(M, \omega)$. Recall that $\mathcal{J}(M, \omega)$ is always contractible. If $X=\mathcal{J}^{\text {int }}(M, \omega) \subset \mathcal{J}(M, \omega)$ is also contractible, then

$$
H_{G}^{*}(X)=H^{*}(B G) .
$$

\section{Rational ruled surfaces}

In this section, we discuss the particular case of rational ruled surfaces, formulating the precise results suggested by the framework of Section 2 .

Symplectic structures. As smooth 4-manifolds, rational ruled surfaces are $S^{2}$-bundles over $S^{2}$. Since $\pi_{2}\left(B \operatorname{Diff}^{+}\left(S^{2}\right)\right) \cong \pi_{2}(B S O(3)) \cong \mathbb{Z} / 2$, there are only two diffeomorphism types classified by the second Stiefel-Whitney class of the bundle (the mod 2 reduction of the Euler class): a trivial $S^{2}$-bundle over $S^{2}$ and a non-trivial $S^{2}$-bundle over $S^{2}$. Since the story for each of these is analogous, we will concentrate here on the trivial bundle, i.e.,

$$
M=S^{2} \times S^{2} .
$$

Symplectic structures on $S^{2} \times S^{2}$ are classified by the following theorem [19].

Theorem 3.1. If $\omega$ is a symplectic form on $S^{2} \times S^{2}$, then it is diffeomorphic to $\lambda \sigma \oplus \mu \sigma$ for some real $\lambda, \mu>0$, where $\sigma$ denotes the standard area form on $S^{2}$ with $\int_{S^{2}} \sigma=1$.

Since the symplectomorphism group and the space of compatible almost complex structures are not affected by positive scalings of the symplectic form and we can switch the two $S^{2}$-factors, it will suffice to consider symplectic structures of the form

$$
\omega_{\lambda}=\lambda \sigma \oplus \sigma \quad \text { with } \quad 1 \leq \lambda \in \mathbb{R} .
$$

From now on, we will use the following notation: $M_{\lambda}=\left(S^{2} \times S^{2}, \omega_{\lambda}\right), 1 \leq$ $\lambda \in \mathbb{R} ; G_{\lambda}=\operatorname{Symp}\left(M_{\lambda}\right)=$ symplectomorphisms of $M_{\lambda} ; \mathcal{J}_{\lambda}=\mathcal{J}\left(M_{\lambda}\right)=$ compatible almost complex structures; $X_{\lambda}=\mathcal{J}^{\text {int }}\left(M_{\lambda}\right)=$ compatible integrable complex structures.

We will also use the following obvious isomorphism:

$$
\begin{aligned}
& H_{2}\left(S^{2} \times S^{2}, \mathbb{Z}\right) \stackrel{\cong}{\longrightarrow} \oplus \mathbb{Z} \\
& m\left[S^{2} \times \mathrm{pt}\right]+n\left[\mathrm{pt} \times S^{2}\right] \longmapsto(m, n) .
\end{aligned}
$$


Compatible integrable complex structures. As a complex manifold, a rational ruled surface is a holomorphic $\mathbb{P}^{1}$-bundle over $\mathbb{P}^{1}$. These are the well-known Hirzebruch surfaces

$$
H_{k}=P(\mathcal{O} \oplus \mathcal{O}(-k)) \quad \text { for some } k \in \mathbb{N}_{0},
$$

where we write $\mathcal{O}(-1)$ for the tautological line bundle over $\mathbb{P}^{1}$ and $P(E)$ for the projectivization of a vector bundle $E$.

Any complex structure $J$ on $S^{2} \times S^{2}$ is isomorphic to $H_{2 k}$ for some $k \in \mathbb{N}_{0}$, while the "odd" Hirzebruch surfaces are diffeomorphic to the non-trivial $S^{2}$-bundle over $S^{2}[\mathbf{2 2}]$. When $\left(S^{2} \times S^{2}, J\right)$ has two embedded $\mathbb{P}^{1}$ 's with self-intersection 0 and themselves intersecting at one point, then $\left(S^{2} \times S^{2}, J\right) \cong H_{0}$. When $\left(S^{2} \times S^{2}, J\right)$ has an embedded $\mathbb{P}^{1}$ with self-intersection $-2 k<0$, then $\left(S^{2} \times S^{2}, J\right) \cong H_{2 k}$.

To understand which of these complex structures $J$ can be made compatible with a symplectic form $\omega_{\lambda}$, for some $1 \leq \lambda \in \mathbb{R}$, it is important to note that the compatibility condition implies that the symplectic form evaluates positively on any $J$-holomorphic curve. Hence, for a compatible $J \in X_{\lambda}$, a homology class $(m, n) \in H_{2}\left(S^{2} \times S^{2} ; \mathbb{Z}\right)$ can only be represented by a $J$-holomorphic curve if $\lambda m+n>0$. This rules out embedded curves with self-intersection less than $-2 \ell$, where $\ell \in \mathbb{N}_{0}$ is such that $\ell<\lambda \leq \ell+1$. In particular, the class $(1,-k) \in H_{2}\left(S^{2} \times S^{2} ; \mathbb{Z}\right)$, with self-intersection $-2 k$, can only be represented by a $J$-holomorphic curve for some $J \in X_{\lambda}$ if $\lambda-k>0$.

This turns out to be the only relevant condition. In fact, we have the following theorem.

Theorem 3.2. Given $1 \leq \lambda \in \mathbb{R}$, there is a stratification of $X_{\lambda}$ of the form

$$
X_{\lambda}=V_{0} \sqcup V_{1} \sqcup \cdots \sqcup V_{\ell},
$$

with $\ell \in \mathbb{N}_{0}$ such that $\ell<\lambda \leq \ell+1$ and where:

(i)

$$
\begin{aligned}
V_{k} \equiv & \left\{J \in X_{\lambda}:\left(S^{2} \times S^{2}\right) \cong H_{2 k}\right\} \\
= & \left\{J \in X_{\lambda}:(1,-k) \in H_{2}(M, \mathbb{Z})\right. \text { is represented } \\
& \text { by a } J \text {-holomorphic sphere }\} .
\end{aligned}
$$

(ii) $V_{0}$ is open and dense in $X_{\lambda}$. For $k \geq 1, V_{k}$ has codimension $4 k-2$ in $X_{\lambda}$.

(iii) $\overline{V_{k}}=V_{k} \sqcup V_{k+1} \sqcup \cdots \sqcup V_{\ell}$.

(iv) For each $k \in \mathbb{N}_{0}$, there is a complex structure $J_{k} \in V_{k}$, unique up to the action of $G_{\lambda}$, for which $g_{\lambda, k} \equiv \omega_{\lambda}\left(\cdot, J_{k} \cdot\right)$ is an extremal Kähler metric.

(v) Denoting by $K_{k}$ the Kähler isometry group of $\left(S^{2} \times S^{2}, \omega_{\lambda}, J_{k}\right)$, we have that

$$
K_{k} \cong \begin{cases}\mathbb{Z} / 2 \ltimes(S O(3) \times S O(3)), & \text { if } k=0 \\ S^{1} \times S O(3), & \text { if } k \geq 1\end{cases}
$$


(vi) Given $J \in V_{k}$, there exists $\varphi \in \operatorname{Diff}\left(S^{2} \times S^{2}\right)$ such that

$$
\left[\varphi^{*}\left(\omega_{\lambda}\right)\right]=\left[\omega_{\lambda}\right] \in H^{2}\left(S^{2} \times S^{2} ; \mathbb{R}\right) \quad \text { and } \quad \varphi_{*}\left(J_{k}\right)=J,
$$

so each stratum $V_{k}$ consists of a unique " $G_{\lambda}^{\mathbb{C}}$ "-orbit, the orbit through $J_{k}$. Moreover, the inclusion

$$
\begin{gathered}
\left(\frac{G_{\lambda}}{K_{k}}\right) \longrightarrow V_{k}=\mathcal{O}_{J_{k}} \\
{[\psi] \longmapsto \psi_{*}\left(J_{k}\right)}
\end{gathered}
$$

is a weak homotopy equivalence.

(vii) Each $V_{k}$ has a tubular neighborhood $N V_{k} \subset X_{\lambda}$, with normal slice given by

$$
H^{1}\left(H_{2 k}, \Theta\right) \cong \mathbb{C}^{2 k-1},
$$

where $\Theta=$ sheaf of holomorphic vector fields on $H_{2 k}$.

(viii) For $k \geq 1$, the representation of $K_{k} \cong S^{1} \times S O(3)$ on the normal slice $\mathbb{C}^{2 k-1}$ at $J_{k} \in V_{k}$ is the following: $S^{1}$ acts diagonally and $S O(3)$ acts irreducibly with highest weight $2(k-1)$.

Proof. Points (i), (ii), (iii), (v) and (vii) follow from standard complex geometry and deformation theory applied to complex structures on $S^{2} \times S^{2}$ [9, I.6]. One needs to check that standard deformation theory can in fact be used here in the context of compatible complex structures. This, together with points (vi) and (viii), will be proved in [2]. Point (iv) is proved in [6].

This theorem shows that the geometric picture suggested by the moment map framework of Section 2 is quite accurate for rational ruled surfaces. It implies, by standard equivariant cohomology theory [2], the following corollary.

Corollary 3.3. Given $\ell \in \mathbb{N}_{0}$ and $\left.\left.\lambda \in\right] \ell, \ell+1\right]$, we have that

$H_{G_{\lambda}}^{*}\left(X_{\lambda} ; \mathbb{Z}\right) \cong H^{*}(B S O(3) \times B S O(3) ; \mathbb{Z}) \oplus \oplus_{k=1}^{\ell} \Sigma^{4 k-2} H^{*}\left(B S^{1} \times B S O(3) ; \mathbb{Z}\right)$, where $\cong$ indicates a group isomorphism.

Compatible almost complex structures. We will now look at the contractible space $\mathcal{J}_{\lambda}$ of compatible almost complex structures on $M_{\lambda}=$ $\left(S^{2} \times S^{2}, \omega_{\lambda}\right)$ for a given $1 \leq \lambda \in \mathbb{R}$. Gromov [15] was the first to study the topology of the symplectomorphism group $G_{\lambda}$ by analyzing its natural action on this space $\mathcal{J}_{\lambda}$. He used pseudo-holomorphic curves techniques and this approach turned out to be quite fruitful $[\mathbf{1}, \mathbf{3}]$. In fact, the action of $G_{\lambda}$ is compatible with a natural geometric stratification of $\mathcal{J}_{\lambda}$, analogous to the one presented in Theorem 3.2 for $X_{\lambda} \subset \mathcal{J}_{\lambda}$. Replacing holomorphic spheres by pseudo-holomorphic ones, and complex deformation theory by gluing techniques for pseudo-holomorphic spheres, one can prove the following theorem $[\mathbf{2 1}]$. 
Theorem 3.4. Given $1 \leq \lambda \in \mathbb{R}$, there is a stratification of the contractible space $\mathcal{J}_{\lambda}$ of the form

$$
\mathcal{J}_{\lambda}=U_{0} \sqcup U_{1} \sqcup \cdots \sqcup U_{\ell},
$$

with $\ell \in \mathbb{N}_{0}$ such that $\ell<\lambda \leq \ell+1$ and where:

(i)

$$
\begin{aligned}
U_{k} \equiv\left\{J \in \mathcal{J}_{\lambda}:(1,-k) \in H_{2}(M ; \mathbb{Z})\right. \text { is represented } \\
\text { by a } J \text {-holomorphic sphere }\} .
\end{aligned}
$$

In particular, $V_{k}=U_{k} \cap X_{\lambda} \subset U_{k}$.

(ii) $U_{0}$ is open and dense in $\mathcal{J}_{\lambda}$. For $k \geq 1, U_{k}$ has codimension $4 k-2$ in $\mathcal{J}_{\lambda}$.

(iii) $\overline{U_{k}}=U_{k} \sqcup U_{k+1} \sqcup \cdots \sqcup U_{\ell}$.

(iv) The inclusion

$$
\begin{aligned}
\left(\frac{G_{\lambda}}{K_{k}}\right) & \longrightarrow U_{k} \\
{[\psi] } & \longmapsto \psi_{*}\left(J_{k}\right)
\end{aligned}
$$

is a weak homotopy equivalence, where $J_{k} \in V_{k} \subset U_{k}$ and $K_{k}=$ Iso $\left(S^{2} \times S^{2}, \omega_{\lambda}, J_{k}\right)$ were characterized in Theorem 3.2. In particular, the inclusion

$$
V_{k} \longrightarrow U_{k}
$$

is also a weak homotopy equivalence.

(v) Each $U_{k}$ has a tubular neighborhood $N U_{k} \subset \mathcal{J}_{\lambda}$ which fibers over $U_{k}$ as a ball bundle.

Contractibility of $\boldsymbol{X}_{\boldsymbol{\lambda}}$. Given $\ell \in \mathbb{N}_{0}$ and $\left.\left.1 \leq \lambda \in\right] \ell, \ell+1\right]$, we can combine the results of Theorems 3.2 and 3.4 to obtain a finite family of diagrams, one for each $0 \leq k \leq \ell$, of the form

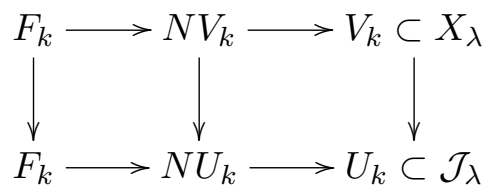

where the vertical arrows are inclusions, the one on the left representing the identity between the fibers of the tubular neighborhoods over $V_{k} \subset U_{k}$. These diagrams are $G_{\lambda}$-equivariant in a suitable sense. Given that $\mathcal{J}_{\lambda}$ is contractible and $V_{k}$ is weakly homotopy equivalent to $U_{k}$, one can use this finite family of diagrams to prove the following theorem $[\mathbf{2}]$.

Theorem 3.5. Given $1 \leq \lambda \in \mathbb{R}$, the space $X_{\lambda}$ of compatible integrable complex structures on $\left(S^{2} \times S^{2}, \omega_{\lambda}\right)$ is weakly contractible.

As far as we know, these are the first known examples of dimension greater than two where the topology of the space of compatible integrable complex structures is understood. 
Cohomology of $B G_{\lambda}$. Theorem 3.5 implies that

$$
H_{G_{\lambda}}^{*}\left(X_{\lambda} ; \mathbb{Z}\right) \cong H^{*}\left(B G_{\lambda} ; \mathbb{Z}\right) .
$$

Combining this isomorphism with Corollary 3.3, we get the following theorem.

Theorem 3.6. Given $\ell \in \mathbb{N}_{0}$ and $\left.\left.\lambda \in\right] \ell, \ell+1\right]$, we have that

$$
\begin{aligned}
H^{*}\left(B G_{\lambda} ; \mathbb{Z}\right) \cong & H^{*}(B S O(3) \times B S O(3) ; \mathbb{Z}) \oplus \\
& \oplus_{k=1}^{\ell} \Sigma^{4 k-2} H^{*}\left(B S^{1} \times B S O(3) ; \mathbb{Z}\right),
\end{aligned}
$$

where $\cong$ indicates a group isomorphism.

Although we used a new point of view, this theorem is not the first result regarding the topology of $B G_{\lambda}$ :

- A formula for the rational cohomology ring of $B G_{\lambda}$ was determined in $[3]$ for any $1 \leq \lambda \in \mathbb{R}$. That formula is incomplete since the relation that determines it is only correct up to "higher order terms" that have to be taken into account. See $[\mathbf{2}]$ for details regarding this issue.

- The integral cohomology ring of $B G_{\lambda}$ was determined for $\lambda=1$ in [15] (where Gromov proves that $G_{1}$ is homotopy equivalent to $S O(3) \times$ $S O(3))$ and for $1<\lambda \leq 2$ in [4].

We hope to include in $[\mathbf{2}]$ a description of the ring structure of $H^{*}\left(B G_{\lambda} ; \mathbb{Z}\right)$ for any $1 \leq \lambda \in \mathbb{R}$.

\section{Symplectomorphisms and complex automorphisms}

Let $(M, \omega)$ be a symplectic manifold and $\mathcal{J}^{\text {int }}(M, \omega)$ the corresponding space of compatible integrable complex structures. The aim of this section is to explain how the condition

$$
\mathcal{J}^{\text {int }}(M, \omega) \sim *
$$

that the space of compatible complex structures be contractible, essentially equates the problem of understanding the topology of $\operatorname{Symp}(M, \omega)$ with the following two problems.

- Understanding the (large-scale) deformation theory of complex structures compatible with $\omega$.

- Understanding the topology of the groups of complex automorphisms of each of these complex structures.

The same is true if one replaces compatible complex structures by tame complex structures in which case the deformation theory in question is the usual deformation theory of Kodaira and Spencer (as the tameness condition is open).

We should point out straight away that we only know condition (1) to hold in a few very simple examples: Riemann surfaces (where the condition holds 
because all almost complex structures are integrable) and simple rational complex surfaces such as $\mathbb{P}^{2}$ and rational ruled surfaces. The work of Lalonde and Pinsonnault [20] suggests that condition (1) also holds for blow-ups of $\mathbb{P}^{2}$ at two points. It would be very interesting to have some understanding of the generality of (1).

The reason why (1) equates the problems above is that it establishes a sort of weak equivalence between two moduli problems - one in symplectic geometry and another in complex geometry. To explain this, we must first recall some basic facts regarding topological groupoids. The reader is referred to $[\mathbf{1 7}$, Section I] for more explanation and details.

Topological groupoids. A topological groupoid is a small topological category in which every morphism is invertible. Thus, a topological groupoid consists of a pair of spaces $(O, M)$ together with some structure maps. $O$ is the space of objects and $M$ the space of isomorphisms between the objects in $O$. The structure maps are $\iota: O \rightarrow M$ assigning the identity isomorphism to each object, two maps $d_{0}, d_{1}: M \rightarrow O$ assigning to a morphism its domain and range, a composition map $\mu: M \times_{O} M \rightarrow M$ and an inverse map $c: M \rightarrow M$ satisfying the obvious identities.

To a groupoid one can associate the space of isomorphism classes $O / I$, which we will call the coarse moduli space, defined as the quotient of $O$ by the equivalence relation generated by $d_{0}(\alpha) \sim d_{1}(\alpha)$. Of course, the groupoid carries much more information than the quotient space.

In many cases, including all those that will concern us, moduli problems can be described in terms of topological groupoids of the following special form:

Example 4.1. Let $G$ be a topological group acting on a space $X$. We define a groupoid $\Gamma(X, G)$ with objects $O=X$ and $M=G \times X$. The structure maps are $\iota(x)=(1, x), d_{0}(g, x)=x, d_{1}(g, x)=g \cdot x, \mu(g, x, h, g \cdot x)=(h g, x)$ and $c(g, x)=\left(g^{-1}, g \cdot x\right)$. The associated quotient space is the space of orbits $X / G$.

Note that $\Gamma(*, G)$ can be naturally identified with the topological group $G$.

Let $\Gamma=(O, M)$ be a topological groupoid, $B$ be a space and $\mathcal{U}=\left\{U_{i}\right\}$ be an open cover of $B$. A 1-cocycle over $\mathcal{U}$ with values in $\Gamma$ is the assignment to each pair $i, j$ of a map

$$
g_{i j}: U_{i} \cap U_{j} \longrightarrow M
$$

satisfying the cocycle condition $\mu\left(g_{i j}, g_{j k}\right)=g_{i k}$ on triple intersections. In particular, $g_{i i}: U_{i} \rightarrow M$ sends each point of $U_{i}$ to an identity morphism and hence amounts to an object selection map $f_{i}: U_{i} \rightarrow O$. The cocycle condition then says that these selections are compatible so that the 1-cocycle represents a continuous family of objects over $B$. One defines equivalence 
between 1-cocycles in the obvious way. An equivalence class of 1-cocycles is called a $\Gamma$-structure on $X$.

Two $\Gamma$-structures $\sigma_{i}, i=0,1$, on $Y$ are said to be concordant $^{1}$ if there is a $\Gamma$-structure $\sigma$ on $Y \times[0,1]$ such that $\sigma_{\mid Y \times 0}=\sigma_{0}$ and $\sigma_{\mid Y \times 1}=\sigma_{1}$. If $\Gamma=\Gamma(*, G)$ is a topological group, concordance is the same as equality, but this is generally not the case.

Any topological groupoid $\Gamma$ has a classifying space $B \Gamma$ which is a homotopy invariant version of the coarse moduli space. It is determined by the equation

$$
[Y, B \Gamma]=\{\text { concordance classes of } \Gamma-\text { structures on } Y\}
$$

In the case of Example 4.1, the classifying space of $\Gamma(X, G)$ is the Borel construction on the $G$-space $X$. That is, if we write $E G$ for a contractible free $G$-space, we have

$$
B \Gamma(X, G) \simeq E G \times{ }_{G} X
$$

In particular, if the $G$-space $X$ consists of a single orbit and $H$ denotes the isotropy subgroup of one of the points of $X$, we have

$$
B \Gamma \simeq \frac{E G \times{ }_{G} G}{H}=\frac{E G}{H} \simeq B H
$$

and so the classifying space of the groupoid is the same as the classifying space of the isotropy group (of any object). If there is more than one orbit, then the map $E G \rightarrow *$ still induces a map

$$
\pi: B \Gamma \longrightarrow \frac{X}{G}
$$

with fibers

$$
\pi^{-1}(G x) \simeq B \operatorname{Aut}(x),
$$

where we have written $\operatorname{Aut}(x)$ for the isotropy group of $x$. Intuitively, this says that the space $B \Gamma$ is obtained by gluing the classifying spaces of the automorphism groups $B \operatorname{Aut}(x)$ via the topology of the moduli space $X / G$, which one can write

$$
B \Gamma \simeq \int_{X / G} B \operatorname{Aut}(x)
$$

In good situations, this is a precise statement [14, Proposition, p. 183]. Even if it is not, it still provides a useful guide to understanding the topology of $B \Gamma$. We will see how this plays out in Examples 4.4 and 4.5 below.

We will say that a map $\phi: \Gamma_{1} \rightarrow \Gamma_{2}$ of topological groupoids is a weak equivalence if the induced map of classifying spaces

$$
B \phi: B \Gamma_{1} \rightarrow B \Gamma_{2}
$$

\footnotetext{
${ }^{1}$ The term used by Haefliger is homotopic.
} 
is a weak homotopy equivalence. In the examples we are concerned with, the source of weak equivalences is the following immediate consequence of the homotopy invariance of the Borel construction:

Lemma 4.2. If $X$ and $Y$ are $G$-spaces and $f: X \rightarrow Y$ is a $G$-equivariant map which is a weak equivalence, then the induced map of groupoids $\Gamma f$ : $\Gamma(X, G) \rightarrow \Gamma(Y, G)$ is a weak equivalence.

Examples of moduli problems. Let $M$ be a compact manifold and $G=$ $\operatorname{Diff}^{+}(M)$ the group of orientation preserving diffeomorphisms of $M$ with the $C^{\infty}$ topology. Consider the following $G$-spaces:

(i) The space $\mathcal{J}^{\text {int }}(M)$ of complex structures on $M$ compatible with some (unspecified) symplectic form on $M$.

(ii) The space $\Omega^{\text {int }}(M)$ of symplectic forms on $M$.

(iii) The space $K^{\text {int }}(M)=\left\{(J, \omega) \in \mathcal{J}^{\text {int }}(M) \times \Omega^{\text {int }}(M): J\right.$ compatible with $\omega\}$ of Kähler structures on $M$.

We will write $\Gamma \mathcal{J}, \Gamma \Omega$ and $\Gamma K$ for the associated topological groupoids. The projections

$$
\mathcal{J}^{\text {int }}(M) \stackrel{\pi_{1}}{\longleftarrow} K^{\text {int }}(M) \stackrel{\pi_{2}}{\longrightarrow} \Omega^{\text {int }}(M)
$$

are $G$-equivariant. The fibers of $\pi_{1}$ are always convex, so $\pi_{1}$ is a weak equivalence. Lemma 4.2 then says that the groupoids $\Gamma \mathcal{J}$ and $\Gamma K$ are weakly equivalent. Condition (1) is precisely the condition that the fibers of $\pi_{2}$ be contractible. If in addition we know that $\pi_{2}$ is surjective, i.e., that all symplectic structures on $M$ are Kähler, then Lemma 4.2 says that $\Gamma \Omega$ is also weakly equivalent to $\Gamma \mathcal{J}$ and $\Gamma K$.

Remark 4.3. We can also consider the non-integrable moduli problems derived from the space $\mathcal{J}(M)$ of almost complex structures compatible with some symplectic form on $M$ and $K(M)$ of almost-Kähler structures. These groupoids are always weakly equivalent to $\Gamma \Omega$. In this light, condition (1) can be seen as the requirement that the inclusions $\mathcal{J}^{\text {int }}(M) \rightarrow \mathcal{J}(M)$ or $K^{i n t}(M) \rightarrow K(M)$ induce weak equivalences of topological groupoids.

Now consider the following variation on the previous situation. Fix a symplectic form $\omega$ on $M$ and consider the orbit

$$
X_{0}=\operatorname{Diff}^{+}(M) \cdot \omega \subset \Omega^{\text {int }}(M) .
$$

Let $X_{1}=\pi_{1}^{-1}\left(X_{0}\right) \subset K^{\text {int }}(M)$ and $X_{2}=\pi_{2}\left(X_{1}\right) \subset \mathcal{J}^{\text {int }}(M)$ so that we have $G$-equivariant maps

$$
X_{0} \stackrel{\pi_{1}}{\longleftarrow} X_{1} \stackrel{\pi_{2}}{\longrightarrow} X_{2}
$$

If (1) holds for the symplectic form $\omega$, then as before all three groupoids are weakly equivalent and using (2) and (3) we have

$$
B \operatorname{Symp}(M, \omega) \simeq B \Gamma\left(X_{0}, G\right) \simeq B \Gamma\left(X_{2}, G\right) \simeq \int_{[J] \in Z} B \operatorname{Aut}(J)
$$


where $Z=X_{2} / \operatorname{Diff}^{+}(M)$ is the coarse moduli space of complex structures compatible with $\omega$, and $\operatorname{Aut}(J)$ is the group of biholomorphisms of the complex manifold $(M, J)$.

Example 4.4 Elliptic curves. Let $M=S^{1} \times S^{1}$ and let $\omega$ be the standard symplectic form. The topology of the diffeomorphism groups of surfaces is well understood. The inclusion of the affine orientation preserving automorphisms of $S^{1} \times S^{1}$ in $\operatorname{Symp}(M, \omega)$ is a weak equivalence, i.e.,

$$
S L_{2}(\mathbb{Z}) \ltimes\left(S^{1} \times S^{1}\right) \simeq \operatorname{Symp}(M, \omega) .
$$

The complex structures on a torus (which are all compatible with any given area form) are parametrized by points in the upper half plane up to the action of $S L(2, \mathbb{Z})$ by fractional linear transformations. Thus the space $Z$ in (4) is obtained from

$$
\Omega=\left\{z \in \mathbb{C}:-\frac{1}{2} \leq \operatorname{Re}(z) \leq \frac{1}{2},|z| \geq 1, \operatorname{Im}(z)>0\right\}
$$

by identifying the boundary points through the maps $z \mapsto z+1$ and $z \mapsto-1 / z$. If $E_{z}$ denotes the elliptic curve parametrized by $z \in \Omega$, we have

$$
\operatorname{Aut}_{\mathbb{C}}\left(E_{z}\right)=\operatorname{Aut}(<1, z>) \ltimes E_{z}
$$

where $\operatorname{Aut}(<1, z>)$ denotes the automorphisms of the lattice generated by $\{1, z\}$. Most lattices have multiplication by -1 as their only non-trivial automorphism. The exceptions are $\langle 1, i>$ which has $\mathbb{Z} / 4$ as automorphism group and $<1, e^{\pi i / 3}>$ which has $\mathbb{Z} / 6$ as automorphism group.

Thus, in this case, (4) says that $B \operatorname{Symp}(M, \omega)$ can be expressed as the double mapping cylinder (or homotopy pushout) of the maps

$$
B\left(\frac{\mathbb{Z}}{4} \ltimes\left(S^{1} \times S^{1}\right)\right) \longleftarrow B\left(\frac{\mathbb{Z}}{2} \ltimes\left(S^{1} \times S^{1}\right)\right) \longrightarrow B\left(\frac{\mathbb{Z}}{6} \ltimes\left(S^{1} \times S^{1}\right)\right)
$$

which amounts to the familiar decomposition of $S L(2, \mathbb{Z})$ as the amalgam of $\mathbb{Z} / 4$ and $\mathbb{Z} / 6$ over $\mathbb{Z} / 2[\mathbf{2 3}, 1.5 .3]$.

Example 4.5. $S^{2} \times S^{2}$. Let us resume Section 3 from the point of view of this section. A theorem of Qin [22] says that any complex structure on $S^{2} \times S^{2}$ is isomorphic to one of the even Hirzebruch surfaces $H_{2 k}$, with $k \geq 0$. Theorem 3.1 (Lalonde-McDuff) says that any symplectic form on $S^{2} \times S^{2}$ is, up to scale, diffeomorphic to $\omega_{\lambda}=\lambda \sigma \oplus \sigma$ for some $\lambda \geq 1$. The complex structures which are compatible with $\omega_{\lambda}$ are the $H_{2 k}$ with $k<\lambda$. So in this example, the coarse moduli space $Z$ is a finite set of points (with a nonHausdorff topology) and (4) expresses $B \operatorname{Symp}\left(M, \omega_{\lambda}\right)$ as a union of spaces with the homotopy type of $B \operatorname{Aut}_{\mathbb{C}}\left(H_{2 k}\right)$ for the allowable values of $k$.

The way the different spaces $B \operatorname{Aut}_{\mathbb{C}}\left(H_{2 k}\right)$ fit together is controlled by the deformation theory of the complex structures, which in this case is the usual Kodaira-Spencer theory. 


\section{References}

[1] M. Abreu, Topology of symplectomorphism groups of $S^{2} \times S^{2}$, Invent. Math. 131 (1998), 1-23.

[2] M. Abreu, G. Granja and N. Kitchloo, Symplectomorphism groups and compatible complex structures on rational ruled surfaces, in preparation.

[3] M. Abreu and D. McDuff, Topology of symplectomorphism groups of rational ruled surfaces, J. Amer. Math. Soc. 13 (2000), 971-1009.

[4] S. Anjos and G. Granja, Homotopy decomposition of a symplectomorphism group of $S^{2} \times S^{2}$, Topology 43 (2004), 599-618.

[5] M.F. Atiyah and R. Bott, The Yang-Mills equations over Riemann surfaces, Phil. Trans. Roy. Soc. Lond. A 308 (1982), 523-615.

[6] E. Calabi, Extremal Kähler metrics, in 'Seminar on differential geometry' (S.T. Yau, ed.), Annals of Math. Studies, 102, Princeton Univ. Press, 1982, 259-290.

[7] E. Calabi, Extremal Kähler metrics II, in 'Differential geometry and complex analysis' (I. Chavel and H.M. Farkas, eds.), Springer-Verlag, 1985, 95-114.

[8] E. Calabi and X.X. Chen, The space of Kähler metrics. II, J. Diff. Geom. 61(2) (2002) 173-193.

[9] F. Catanese, Moduli of algebraic surfaces, Lecture Notes Math., 1337, Springer, 1988, $1-83$.

[10] S. Donaldson, Remarks on gauge theory, complex geometry and 4-manifold topology, in 'Fields medallists' lectures' (M.F. Atiyah and D. Iagolnitzer, eds.), World Sci., 1997, 384-403.

[11] S. Donaldson, Symmetric spaces, Kähler geometry and Hamiltonian dynamics, in 'Northern California Symplectic Geometry Seminar' (Y. Eliashberg et al., eds.), American Mathematical Society, 1999, 13-33.

[12] S. Donaldson, Scalar curvature and projective embeddings I, J. Diff. Geom. 59 (2001), 479-522.

[13] S. Donaldson, Scalar curvature and stability of toric varieties, J. Diff. Geom. 62 (2002), 289-349.

[14] E. Dror-Farjoun, Cellular spaces, null spaces and homotopy localization, Lecture Notes Math., 1622, Springer, 1996.

[15] M. Gromov, Pseudo holomorphic curves in symplectic manifolds, Invent. Math. 82 (1985), 307-347.

[16] A. Hwang, On the Calabi energy of extremal Kähler metrics, Int. J. Math. 6 (1995), $825-830$.

[17] A. Haefliger, Homotopy and integrability, Lecture Notes Math., 197, Springer, 1971, 133-163.

[18] F. Kirwan, Cohomology of quotients in symplectic and algebraic geometry, Math. Notes 31, Princeton Univ. Press, 1984.

[19] F. Lalonde and D. McDuff, J-holomorphic spheres and the classification of rational and ruled symplectic 4-manifolds, in 'Contact and symplectic geometry' (C. Thomas, ed.), Cambridge University Press, 1996, 3-42.

[20] F. Lalonde and M. Pinsonnault, The topology of the space of symplectic balls in rational 4-manifolds, Duke Math. J. 122 (2004), 347-397.

[21] D. McDuff, Almost complex structures on $S^{2} \times S^{2}$, Duke Math. J. 101 (2000), 135-177. 
[22] Z. Qin, Complex structures on certain differentiable 4-manifolds, Topology 32 (1993), $551-566$.

[23] J.-P. Serre, Trees, in 'Springer Monographs in Mathematics,' Springer-Verlag, Berlin, $2003, \mathrm{x}+142$.

[24] C.L. Siegel, Symplectic geometry, Academic Press, 1964.

Departamento de Matemática

Instituto Superior TÉCNICO

Av. Rovisco PAIS

1049-001 Lisboa, Portugal

E-mail address: mabreu@math.ist.utl.pt,ggranja@math.ist.utl.pt

Department of Mathematics

UNIVERSITY OF CALIFORNIA

SAN DIEGO

E-mail address: nitu@math.ucsd.edu

Received 02/21/2005. Nitu Kitchloo is supported in part by NSF through grant DMS 0436600. Miguel Abreu and Gustavo Granja are supported in part by FCT through program POCTI-Research Units Pluriannual Funding Program and grants POCTI/MAT/57888/2004 and POCTI/MAT/58497/2004.

Miguel Abreu and Gustavo Granja are members of the Center for Mathematical Analysis, Geometry and Dynamical Systems, which supported visits of Nitu Kitchloo to ISTLisbon. We thank the Center for its research environment and hospitality. Miguel Abreu also thanks the organizers of the Conference on Symplectic Topology at Stare Jablonki, Poland, for the opportunity to speak about this work at such a wonderful event. 